\title{
Interactions between perceptual organization based on Gestalt laws and those based on hierarchical processing
}

\author{
SHIHUI HAN \\ University of Science and Technology of China, Beijing, China \\ University of California, Davis, Califormia \\ and VA Northern California System of Clinics, Martinez, Califormia \\ and \\ GLYN W. HUMPHREYS \\ University of Birmingham, Birmingham, England
}

\begin{abstract}
Our recent research with compound stimuli (Han, Humphreys, \& Chen, 1999a) suggests that grouping between local elements facilitates the perception of global structure, whereas encoding closure in local elements enhances their segmentation. The present study presents further evidence supporting this assertion. Experiment 1 first developed a new paradigm in which grouping between local elements was manipulated. Subjects responded to the orientations of perceptual groups consisting of local arrows or triangles embedded in background crosses. Responses to the orientations of the groups were slowed as a function of the increased contrast of the crosses, indicating that the strength of grouping between local arrows or triangles was gradually weakened by increasing the contrast of the crosses. Using a similar paradigm, Experiments 2 and 3 investigated the role of Gestalt factors in hierarchical analysis. Global arrows or triangles made up of local arrows, or triangles were embedded in background crosses. Subjects responded to global or local stimuli in terms of orientation or closure. Increasing the contrast of the background crosses produced stronger effects on global responses than on local responses and resulted in elimination of the global precedence effect and emerging of a local precedence effect, which was stronger for closure discrimination than for orientation discrimination. These results provide new evidence supporting our previous claim about the role of Gestalt factors in hierarchical analysis.
\end{abstract}

The Gestalt psychologists (e.g., Wertheimer, 1923) emphasized the role of perceptual organization in vision and maintained that early stages of vision act to segment the visual field into perceptual groups or objects on the basis of the principles of perceptual grouping, such as proximity, similarity, and closure. Recent psychological evidence supports this proposal, suggesting that perceptual grouping can operate preattentively (Baylis \& Driver, 1993; Humphreys, Olson, Romani, \& Riddoch, 1996; Kahneman \& Henik, 1981; Kahneman \& Treisman, 1984; Kramer \& Jacobson, 1991; Moore \& Egeth, 1997), though different factors dominate perceptual grouping across different time courses (e.g., proximity operates prior to

This study was supported by the National Foundation of Sciences of China; the Human Frontier Science Programme Organization, the Biology and Biotechnology Research Council, and the Medical Research Council (U.K.); and the National Institute of Mental Health (MH41544 ), the National Institute of Neurological Disease and Stroke (NS32893), and the VA Research Service. Correspondence should be addressed to S. Han, Research Service (151), Veterans Affairs Medical Center, NCSC, 150 Muir Rd., Martinez, CA 94553 (e-mail: shan@) ebire.org).

Accepted by previous editor, Myron L. Braunstein shape similarity; similarity based on closure is computed prior to similarity based on orientation and so forth; BenAv \& Sagi, 1995; Chen, 1982a, 1986).

Visual processing over time may also be differentially sensitive to the hierarchical structure of stimuli. Using compound stimuli, in which global letters were made up of local ones, Navon (1977) found that reaction times (RTs) for global letters were faster than those for local ones (i.e., there was a global RT advantage) and that the global level interfered with responses to the local level when letters at the two levels were inconsistent (i.e., there was global interference); in contrast, the local level had no effect on responses to global letters. On the basis of these findings, Navon put forward a global precedence hypothesis, proposing that global properties are extracted from visual arrays and processed earlier than local properties.

Subsequent studies have shown that several factors interact with the processing dominance of global levels of compound stimuli. For example, the global RT advantage can be eliminated by increasing the overall visual angle of the stimuli (Kinchla \& Wolfe, 1979; Lamb \& Robertson, 1990), reducing the number of local letters (LaGasse, 1993; Martin, 1979; Navon, 1983), and removing lowspatial-frequency components from the compound stim- 
uli (Badcock, Whitworth, Badcock, \& Lovegrove, 1990; Hughes, Fendrich, \& Reuter-Lorenz, 1990; Lamb \& Yund, 1993, 1996; though see Hübner, 1997). The global RT advantage is also affected by where stimuli appear on the retina (Grice, Canham, \& Boroughs, 1983; Pomerantz, 1983) and spatial uncertainty concerning where the stimuli will appear (Lamb \& Robertson, 1988). Global interference has also been found to be influenced by display durations (Luna, 1993; Luna, Marcos-Ruiz, \& Merino, 1995; Paquet \& Merikle, 1984): There is stronger global-to-local interference at shorter exposure durations than at longer exposure durations.

What is the relationship between perceptual organization based on Gestalt factors and that based on the levels of hierarchical structures? Kimchi (1994) reported an interaction between hierarchical pattern analysis and the processing of closure. She had subjects classify global or local stimuli in terms of their orientation and closure and found that the global RT advantage was observed only for classifications based on orientation but not for those based on closure. Kimchi argued that the visual system is tuned to detect rapidly configural properties such as closure, irrespective of the hierarchical analysis of patterns. It may also be that global processing requires focal attention. Enns and Kingstone (1995) had subjects search for a target feature (e.g., triangle vs. square) among distractors made up of compound patterns. The target was equally likely to occur at the local level, the global level, or at both levels of the compounds. Enns and Kingstone found larger search slopes for the global targets than for the local targets. From this, they suggested that the perception of the global structure in a compound stimulus requires an attention-demanding grouping operation, relative to the processes needed for the perception of the local elements.

Our recent work (Han, Humphreys, \& Chen, 1999a) has further revealed interactions between different Gestalt factors in hierarchical analysis. We presented global arrows or triangles composed of local arrows or triangles either on a blank background (where local elements grouped strongly by proximity, similarity, and/or continuity) or on a background of crosses (where similarity of shapes dominated local element grouping, but where, on the basis of proximity, target and background elements could not be distinguished; likewise grouping by similarity of luminance was also weakened). Subjects responded to global or local stimuli in terms of orientation or closure. We found that there were global precedence effects for both the orientation discrimination and the closure discrimination tasks; however, the effects were weaker for closure discrimination. We suggested that segmentation is facilitated and that less local selection effort is engaged in the closure discrimination task than in the orientation discrimination task, making the global precedence and interference effects weaker. We also found that the saliency of the global shape decreased and a local advantage emerged, particularly for the closure discrimination task, when compound stimuli were presented among the background crosses. We argued that back- ground crosses forced global shapes to be formed by shape similarity (i.e., the local elements of hierarchical patterns were identical to each other but different from the crosses in shape), rather than by proximity grouping. Because studies have demonstrated that grouping by similarity of shapes occurs later in time or is perceived slower than grouping by proximity (Ben-Av \& Sagi, 1995; Chen, 1982a, 1986; Han, Humphreys, \& Chen, 1999b), our data suggest that weakening perceptual grouping of local elements of hierarchical patterns eliminates the perception of the global structure of hierarchical patterns.

In the present study, we provided further evidence supporting our proposal that weakening the grouping of local elements into global forms produces a stronger effect on the global perception than on the local perception and that perceptual grouping of local elements is crucial for the perception of global structure of hierarchical patterns. In Experiment 1, we introduced a new paradigm in which we manipulated the contrast of background figures to reduce grouping between local elements. Subjects were required to respond to the orientations of groups made up of local arrows or triangles that were embedded against a background of crosses (see Figure 1). The arrows or triangles were arranged in arrays giving rise to two possible perceptual organizations: either vertical columns or horizontal rows. In one condition, the groups composed of local arrows or triangles were presented alone (i.e., the background crosses were invisible). The perceptual organization of the arrows or triangles was determined by the relative distance between columns or rows - that is, the interelement spacing (proximity) dominated perceptual organization. When the background crosses were presented, all the local elements of the stimulus (including the arrows, triangles, and crosses) were equidistant. Under this condition, proximity could no longer dominate perceptual organization. When the contrast of the crosses was lower than that of the arrows or triangles, the arrows or triangles in columns or rows were identical to each other and different from the crosses in contrast (or luminance) and shape. Therefore, similarity of contrast (or luminance) and shape dominated perceptual organization. Progressively increasing the contrast of the crosses to the same level of that of the arrows or triangles reduced the role of similarity of contrast (luminance) in grouping the arrows or triangles into columns or rows and made similarity of shape become dominant. Since grouping by similarity of shapes occurs later in time or is perceived slower than grouping by proximity (Ben-Av \& Sagi, 1995; Chen, 1982a, 1986; Han et al., 1999b), gradually increasing the contrast of the crosses should weaken perceptual grouping of local arrows or triangles. We measured RTs to the orientations of the groups composed of the local arrows or triangles as a function of the contrast of the background crosses in order to examine how elimination of proximity grouping and enhancement of grouping by similarity of shape affected subjects' response speed.

The same procedure to manipulate perceptual grouping of local elements was then used in Experiments 2 and 
3 in which subjects discriminated the global or local shapes of compound patterns (i.e., global arrows or triangles composed of local arrows or triangles) embedded in a background of crosses. Increasing the contrast of the background crosses should progressively weaken local element grouping by proximity and by similarity of contrast and should strengthen local element grouping by similarity of shape. We assessed whether this effect was stronger on the perception of global structure than on the perception of local elements, measured in terms of both relative RT advantage and interference effects between the global and local levels of hierarchical patterns. Furthermore, since previous work suggests that the selection of local elements is easier in closure discrimination tasks than in orientation discrimination tasks (Han \& Humphreys, 1999; Han et al., 1999a), responses to local elements should be more robust to effects of the background crosses (which may group by proximity with or laterally mask local elements; see Podrouzek, Modigliani, \& Di Lollo, 1992) in the closure discrimination task than in the orientation discrimination task. Thus, our proposal that less local selection effort is engaged in the closure discrimination task than in the orientation discrimination task will be supported by any findings that increasing the contrast of the background crosses produces a stronger effect on local RTs when the task requires discrimination of orientation than when the task requires discrimination of closure.

One further question addressed here is how variations in the strength of local element grouping affects the interference effect between global and local levels of compound stimuli. Previous research has shown that overall RT advantages and interference effects may not covary (Lamb \& Robertson, 1988; Navon \& Norman, 1983). For instance, eliminating low-spatial-frequency components in compound stimuli reduces the global RT advantage but does not alter the interference effect (Lamb \& Yund, 1993, 1996). These findings suggest that the overall RT advantages and any interference effects do not reflect order of processing in a simple way and may be produced by different mechanisms (May, Gutierrez, \& Harsin, 1995). In the present study, we evaluated the relationship between any overall RT advantage and interference effects between local and global levels of compound stimuli as a function of the strength of local element grouping.

\section{EXPERIMENT 1}

One traditional method to study grouping effects is to measure responses to global orientations of patterns formed by perceptual groups (Ben-Av \& Sagi, 1995; Kurylo, 1997). Here, we adopted this procedure to assess how the presence of the background crosses affected grouping between the local arrows or triangles, measuring RTs to the orientations of the groups. Because the local arrows are different from the crosses in orientation and the local triangles are different from the crosses in closure, we were also interested to see whether effects emerged of closure and orientation on perceptual group- ing. Because previous studies suggest that similarity of closure is perceived prior to (or faster than) similarity of orientation (Chen, 1982a, 1986; Han et al., 1999b), stronger effects of the crosses on stimuli composed of local arrows than those on stimuli composed of local triangles will provide evidence for this assertion.

\section{Method}

Subjects. Fourteen undergraduate students (4 male, 10 female; 19-2l years of age) from the University of Science and Technology of China participated in Experiment 1 as paid volunteers. All had normal or corrected-to-normal vision.

Apparatus. Data collection and stimulus presentation were controlled by a 486 personal computer. Stimuli were presented on a 21 in. NEC Multisync 3-D color monitor at a viewing distance of about $70 \mathrm{~cm}$.

Stimuli. Stimuli consisted of square arrays of local arrows or triangles and crosses arranged in an $11 \times 11$ matrix, as illustrated in Figure 1. The local elements appeared as black on a white background. The local arrows or triangles were arranged so that they were in columns or rows separated by the crosses. The global array was $5.3 \times 5.9 \mathrm{~cm}$, and the local figure was $0.3 \times 0.4 \mathrm{~cm}$. The global array and each local figure subtended visual angles of $4.3^{\circ} \times 4.8^{\circ}$ and $0.25^{\circ} \times 0.33^{\circ}$, respectively. Each of the crosses had the same horizontal and vertical size as that of each local arrow or triangle. The distance between adjacent triangles or arrows was equivalent to that between each triangle or arrow and its neighboring crosses.

The RGB values for the background screen were $25,25,25$. The RGB values for the arrows or triangles were $5,5,5$. The RGB values for the arrows and triangles and the background screen were kept unchanged. The RGB values of the crosses were manipulated in five conditions. Under each of the five conditions, the RGB values were the same (i.e., 25, 22, 18, 12, and 5), and the relevant uniform intensities of background with these RGB values were 45.5 , $34.5,22.3,8.6$, and $1.1 \mathrm{~cd} / \mathrm{m}^{2}$. As the RGB values decreased, the contrast between the crosses and background screen increased from 0.000 (Condition C1), 0.0638 (Condition C2), 0.1628 (Condition C3), 0.3514 (Condition C4), to 0.6667 (Condition C5). This manipulation made the contrast of the crosses increasingly similar to that of the arrows and triangles; thus, the range of the contrast ran from matching the white background (thus rendering them invisible) to those that matched the target elements. Accordingly, proximity grouping between arrows or between triangles, based on elements having equal contrast, decreased. Meanwhile, grouping by similarity of luminance was also weakened because the difference in luminance between local arrows (or triangles) and the white background was greater than that between target elements and the background crosses. This meant that grouping based on shape similarity became more important as the contrast of the crosses increased.

Procedure. The subjects were required to discriminate horizontal and vertical orientations of the groups composed of arrows or triangles regardless of the change of the contrast of the crosses. Each trial began with a 1,000 -msec warning beep and the presentation of a small black dot at the center of the screen as fixation. After another 1,000 msec, the fixation was overlapped by the stimulus display that was presented at the center of the screen and stayed on until the subjects responded. There were three blocks of 80 trials after 24 practice trials. The subjects responded to the orientations of the groups composed of the arrows or triangles by pressing one of two keys on a standard keyboard with the right and left middle fingers. The relationship between the orientations of the groups and the responding hand was counterbalanced across subjects. Instructions stressed both accuracy and speed.

RTs and error rates were subjected to a repeated measures analysis of variance (ANOVA), with two factors being the contrast of the crosses and the shapes of the local elements making up the groups (arrows vs. triangles) 

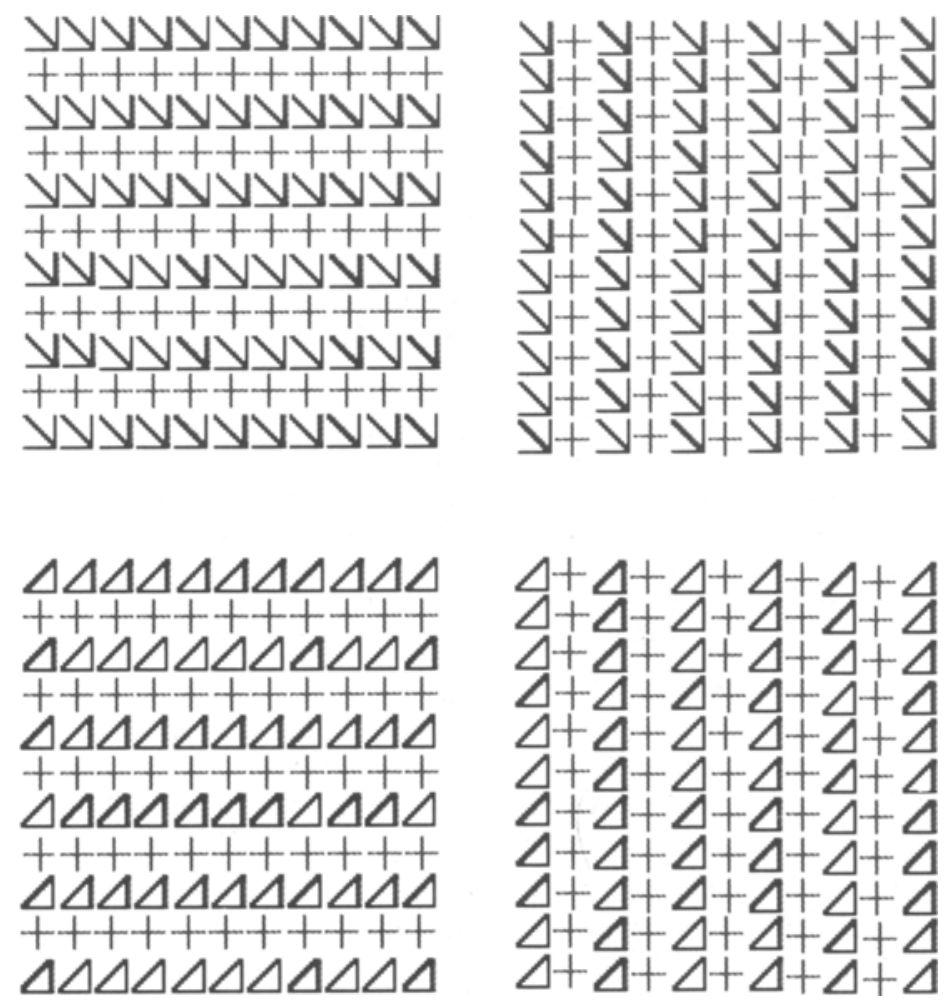

Figure 1. Four stimulus patterns used in Experiment 1.

\section{Results}

The mean percentages of errors for discriminating the orientations of the groups composed of the arrows and the triangles were $5.3 \%$ and $4.4 \%$, respectively. The effects of contrast and shapes of local elements and their interactions were not significant $(p>.1)$. Comparisons between the error rates and the mean RTs indicated that there was no speed-accuracy tradeoff. The mean error rates under each condition are given in Table 1 .

The mean RTs for correct responses to the orientations of the groups made up of local arrows or triangles are shown in Table 2. An ANOVA indicated a main effect of contrast $[F(4,52)=7.82, p<.0005]$; RTs were slowed as the contrast of the crosses increased. The effect of the shapes of the local elements was not significant $(p>.5)$. However, the interaction between these two factors reached significance $[F(4,52)=3.39, p<.015]$,

Table 1

Mean Percent Errors and Standard Errors

Under Each Condition of Experiment 1

\begin{tabular}{|c|c|c|c|c|}
\hline \multirow{3}{*}{$\begin{array}{c}\text { Contrast } \\
\text { Condition }\end{array}$} & \multicolumn{4}{|c|}{ Local Shape } \\
\hline & \multicolumn{2}{|c|}{ Arrow } & \multicolumn{2}{|c|}{ Triangle } \\
\hline & $M$ & $S E$ & $M$ & $S E$ \\
\hline $\mathrm{Cl}$ & 4.8 & 1.8 & 4.8 & 2.0 \\
\hline $\mathrm{C} 2$ & 5.2 & 1.8 & 3.0 & 1.3 \\
\hline C3 & 3.6 & 1.7 & 4.0 & 1.5 \\
\hline $\mathrm{C} 4$ & 7.6 & 2.9 & 4.4 & 1.0 \\
\hline C5 & 5.5 & 1.6 & 5.9 & 1.9 \\
\hline
\end{tabular}

reflecting the fact that RTs were slower to the orientations of groups made up of local arrows than to those made up of local triangles when the contrast of the background crosses was high. Separate analyses showed that the effect of contrast was significant for the stimuli made up of local arrows $[F(4,52)=19.58, p<.0005]$ but not for those made up of local triangles $(p>.3)$. For the stimuli made up of local arrows, RTs in Condition $\mathrm{C} 1$ did not differ from those in Condition C2 $[t(13)=0.19, p>.8]$, RTs in Condition C2 were faster than those in Condition $\mathrm{C} 3[t(13)=3.75, p<.003]$, RTs in Condition C3 did not differ from those in Condition $\mathrm{C} 4[t(13)=0.93$, $p>.6]$, and RTs in Condition C4 were faster than those in Condition C5 $[t(13)=4.19, p<.001]$.

\section{Discussion}

RT was used as an index reflecting the speed of grouping. We found that increasing the contrast of the background crosses reduced RTs to the orientations of the groups composed of the local elements embedded in the background crosses. This dynamic variation of the RTs as a function of the contrast of the crosses indicates that grouping spatially separate elements into a unified whole is gradually slowed by increasing the contrast of the crosses. Interestingly, the effect of increasing the contrast of the crosses was significant only for the stimuli composed of the local arrows, but not for the stimuli composed of the local triangles. The pattern of the RT results was expected given that grouping by proximity oc- 
Table 2

Mean Reaction Times (in Milliseconds) and Standard Errors Under Each Condition of Experiment 1

\begin{tabular}{ccccc} 
& \multicolumn{4}{c}{ Local Shape } \\
\cline { 2 - 5 } Contrast & \multicolumn{2}{c}{ Arrow } & \multicolumn{2}{c}{ Triangle } \\
\cline { 2 - 5 } Condition & $M$ & $S E$ & $M$ & $S E$ \\
\hline C1 & 519 & 14.9 & 524 & 15.5 \\
C2 & 518 & 15.4 & 533 & 18.1 \\
C3 & 539 & 17.4 & 543 & 16.2 \\
C4 & 545 & 15.7 & 530 & 21.1 \\
C5 & 570 & 17.4 & 543 & 16.4 \\
\hline
\end{tabular}

curs earlier or is perceived faster than grouping by similarity of shapes and given that similarity grouping based on closure is encoded prior to that based on orientation (Ben-Av \& Sagi, 1995; Chen, 1982a, 1986; Han et al., 1999 b). Proximity grouping of local arrows should be gradually eliminated when the contrast of the crosses is progressively increased, and these stimuli may then group by similarity of orientation. Responses to the stimuli were then slowed, presumably because grouping by local orientations occurred slowly or at a late stage. However, despite the elimination of proximity grouping as the contrast of the crosses increased, the grouping of local triangles was still efficient, presumably because it was based on an early and fast factor, similarity of closure (Chen, 1982a, 1986; Han et al., 1999b). As a consequence, responses to triangles were less affected by variations in the contrast of the crosses.

We should also note that spatial-frequency noise may also have been introduced when the contrast of the background crosses increased, and this may have impaired the process of grouping (Ginsburg, 1986). However, the manipulation of the contrast of the background crosses was the same for the two types of stimuli (local arrows or triangles), and so any spatial frequency noise introduced by the crosses would be the same. If the effect of spatial frequency noise was crucial, RTs should be slowed regardless of the type of grouping involved (i.e., grouping by similarity of closure or grouping by similarity of orientation). Our data gave little support to this argument.

\section{EXPERIMENT 2}

Experiment 1 demonstrated that the background crosses affected grouping of local elements. Increasing the contrast of the crosses progressively eliminated grouping by proximity and similarity of luminance of local elements and made similarity of shape dominate grouping. Because grouping by similarity of shape occurs slowly or at a late stage of perception (Ben-Av \& Sagi, 1995; Chen, 1982a, 1986; Han et al., 1999b), grouping of local elements was progressively weakened by the increase of the contrast of the background crosses, indexed by slowed responses to orientations of the groups. This procedure to weaken perceptual grouping by manipulating the contrast of the background crosses was employed in Experiment 2 in order to study the role of perceptual grouping in the processing of hierarchical patterns. Because our previous study (Han et al., 1999a) suggested that perceptual grouping of local elements of hierarchical stimuli is crucial for the perception of global structure, we expected weakening grouping between local elements to retard the processing of global properties and thus eliminate the global precedence effect. Hierarchical patterns (i.e., global arrows made up of local arrows) were used in Experiment 2. Subjects made judgments based on orientation of the global or local arrows. The hierarchical stimuli were embedded in a background of crosses. The contrast of the crosses was manipulated as that in Experiment 1 . Dynamic variation of the relevant advantage of global and local processing and their interference should provide strong evidence for how the strength of grouping between local elements affects the global precedence effect.

\section{Method}

Subjects. Nine undergraduate and graduate students (all male; 21-24 years of age) from the University of Science and Technology of China participated in Experiment 2. All had normal or correctedto-normal vision.

Apparatus, Stimuli, and Procedure. All aspects were the same as those in Experiment 1 except for the following. Compound stimuli were used, as shown in Figure 2. Each compound stimulus consisted of global arrows made up of local arrows directing down right or up right. Shapes at the global and local levels were either consistent or inconsistent. The local arrows were arranged in an $8 \times 8$ matrix. The global figure was $3.8 \times 4.4 \mathrm{~cm}$, subtending a visual angle of $3.1^{\circ} \times 3.6^{\circ}$. The whole pattern including background crosses was $4.8 \times 5.6 \mathrm{~cm}$, subtending a visual angle of $3.9^{\circ} \times 4.6^{\circ}$.

A selective-attention paradigm was used. The subjects were required to discriminate orientations of global or local arrows, regardless of changes in the contrast of the background crosses. There were four blocks each for the globally and locally relevant tasks. Each block of 60 trials was presented after 16 practice trials. The contrast manipulation was randomized across trials, and the presentation sequence of the global and local blocks was also randomized for each subject. The subjects responded to the global or local figures by pressing one of two keys on a standard keyboard with the right and left middle fingers. The relationship between the stimuli and the responding hand was counterbalanced across subjects.

RTs and error rates were subjected to a repeated measures ANOVA, with three factors: contrast of the background crosses, globality (discriminating targets at the global or local levels), and consistency (whether the global and local shapes were consistent or inconsistent).

\section{Results}

The mean percentage errors for the globally and locally relevant tasks were $1.8 \%$ and $2.2 \%$, respectively. The mean error rates under each condition are given in Table 3. An ANOVA indicated only a reliable main effect of consistency $[F(1,8)=7.25, p<.025]$. The error rates were higher in the inconsistent condition than in the consistent condition.

The mean global and local RTs are presented in Table 4 as a function of the contrast of the background crosses. An ANOVA indicated a reliable main effect of contrast $[F(4,32)=61.48, p<.0005]$; RTs were slowed when the contrast of the crosses increased. There was also a main effect of consistency $[F(1,8)=225.02, p<.0005]$; RTs were faster when the global and local shapes were consis- 

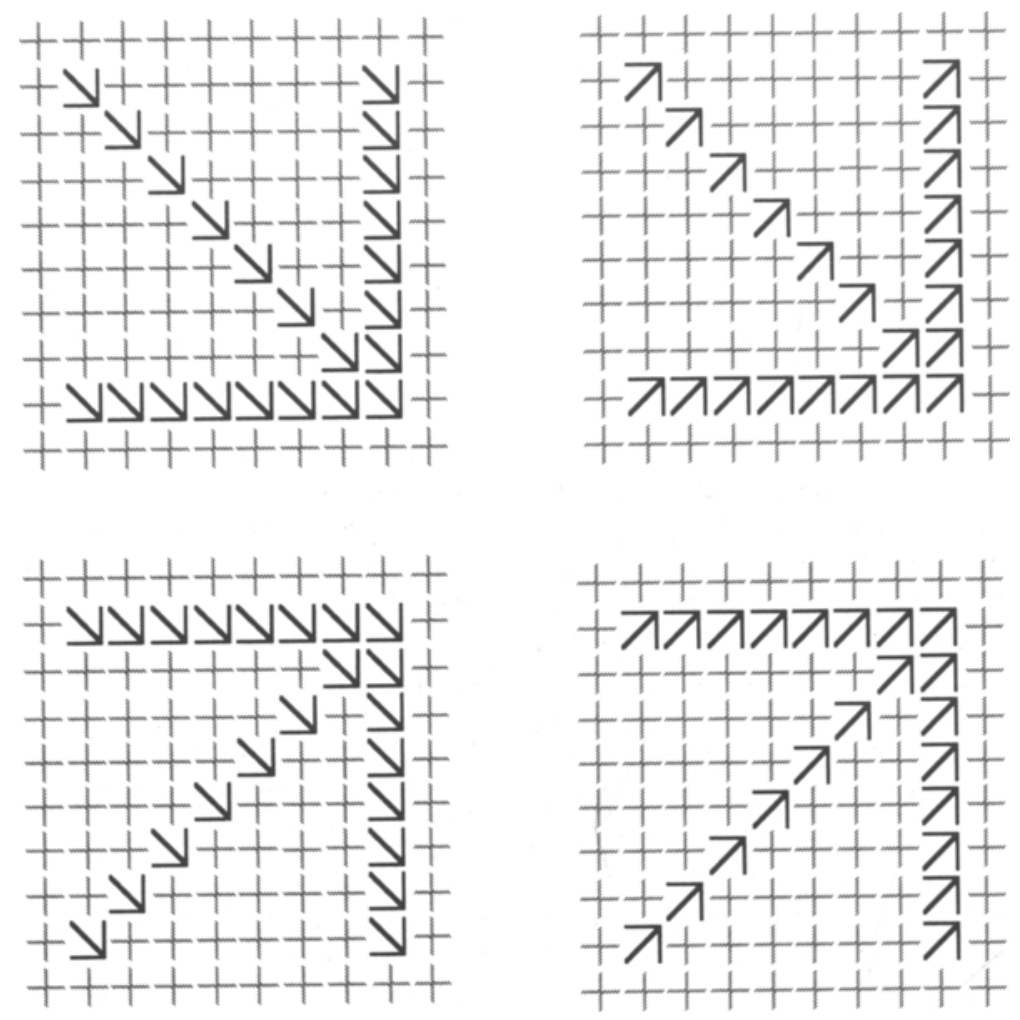

Figure 2. Compound stimuli used in Experiment 2. Global arrows pointing down right or up right are composed of local arrows pointing down right or up right.

tent than when they were inconsistent. The effect of globality was marginally significant $[F(1,8)=4.36, p<.07]$.

There was a significant contrast $\times$ globality interaction $[F(4,32)=21.28, p<.0005]$, reflecting the fact that the effect of contrast variation was greater on the global RTs than on the local RTs. A further orthogonal planned contrast test indicated that increasing the contrast of the crosses slowed responses to both the global stimuli $[F(4,32)=60.18, p<.0005]$ and the local stimuli $[F(4,32)=3.76, p<.02]$. The interactions between globality and consistency $[F(1,8)=1.20, p>.3]$ and between consistency and contrast $[F(4,32)=1.45, p>.2]$ did not reach significance. However, as the contrast of the crosses increased, the global interference effect decreased and was replaced by a local interference effect, as shown

Table 3

Mean Percent Errors and Standard Errors Under Each Condition of Experiment 2

\section{Global Local}

\begin{tabular}{|c|c|c|c|c|c|c|c|c|}
\hline \multirow{3}{*}{$\begin{array}{l}\text { Contrast } \\
\text { Condition }\end{array}$} & & \\
\hline & \multicolumn{2}{|c|}{ Consistent } & \multicolumn{2}{|c|}{ Inconsistent } & \multicolumn{2}{|c|}{ Consistent } & \multicolumn{2}{|c|}{ Inconsisten } \\
\hline & $M$ & $\overline{S E}$ & $M$ & $S E$ & $\bar{M}$ & $S E$ & $M$ & $S E$ \\
\hline $\mathrm{Cl}$ & 0.6 & 0.6 & 1.7 & 0.9 & & 1. & 1.7 & 0.8 \\
\hline $\mathrm{C} 2$ & 1. & 0.7 & 0.6 & 0.6 & & 0. & 3.6 & 1.6 \\
\hline $\mathrm{C} 3$ & 1.6 & 1.1 & 2.7 & 1.1 & 1.1 & 0.7 & 3.2 & 1.1 \\
\hline C4 & 1.6 & 1.1 & 2.2 & 0.9 & 0.6 & 0.6 & 2.8 & 0.9 \\
\hline $\mathrm{C} 5$ & 0.6 & 0.6 & 5.5 & 2.1 & 2.2 & 0.9 & 4.1 & 1.2 \\
\hline
\end{tabular}

in Figure 3. This was demonstrated by the significant contrast $\times$ globality $\times$ consistency interaction $[F(4,32)=$ $22.28, p<.001]$.

Separate analysis for each contrast condition showed that global RTs were faster than local RTs for Conditions $\mathrm{C} 1[F(1,8)=38.07, p<.0005]$ and $\mathrm{C} 2[F(1,8)=$ $18.72, p<.003]$. There was no difference between global and local RTs for Conditions C3 $[F(1,8)=4.08, p>.08]$ and $\mathrm{C} 4[F(1,8)=1.79, p>.2]$. Local RTs were faster than global RTs for Condition C5 $[F(1,8)=5.30, p<$ $.05]$. The effect of consistency was significant under all the contrast conditions $(p<.006)$. The globality $\times$ consistency interaction was significant (but in opposite directions) for Conditions $\mathrm{Cl}[F(1,8)=14.32, p<.005], \mathrm{C} 4$ $[F(1,8)=15.74, p<.004]$, and $\mathrm{C} 5[F(1,8)=12.58, p<$ $.008]$; it was not significant for Conditions $\mathrm{C} 2[F(1,8)=$ $1.89, p>.2]$ and $\mathrm{C} 3[F(1,8)=1.14, p>.3]$.

\section{Discussion}

The data of Experiment 2 show a systematic variation of the relative advantage of global and local processing of hierarchical patterns as a function of the contrast of the background crosses. In Condition $\mathrm{Cl}$ in which there were no background crosses, a global precedence effect was found. Global RTs were faster than local RTs, and the global interference on local responses was stronger than the reverse. In Condition $\mathrm{C} 2$ in which the background 
Table 4

Mean Reaction Times (in Milliseconds) and Standard Errors Under Each Condition of Experiment 2

\begin{tabular}{|c|c|c|c|c|c|c|c|c|}
\hline \multirow{3}{*}{$\begin{array}{l}\text { Contrast } \\
\text { Condition }\end{array}$} & \multicolumn{4}{|c|}{ Global } & \multicolumn{4}{|c|}{ Local } \\
\hline & \multicolumn{2}{|c|}{ Consistent } & \multicolumn{2}{|c|}{ Inconsistent } & \multicolumn{2}{|c|}{ Consistent } & \multicolumn{2}{|c|}{ Inconsistent } \\
\hline & $M$ & $S E$ & $M$ & $S E$ & $M$ & $S E$ & $M$ & $S E$ \\
\hline $\mathrm{Cl}$ & 457 & 16.5 & 480 & 11.3 & 485 & 16.3 & 551 & 16.8 \\
\hline $\mathrm{C} 2$ & 465 & 14.5 & 487 & 13.2 & 500 & 15.2 & 545 & \\
\hline $\mathrm{C} 3$ & 491 & 13.2 & 526 & 16.4 & 521 & 17.8 & 538 & 18.1 \\
\hline $\mathrm{C} 4$ & 509 & 17.9 & 579 & 18.2 & 525 & 17.7 & 535 & 16. \\
\hline $\mathrm{C} 5$ & 525 & 15.5 & 600 & 19.5 & 531 & 18.4 & 548 & 15.7 \\
\hline
\end{tabular}

crosses were presented with low contrast, global RTs were still faster than local ones, but global interference was no more stronger than local interference. In Condition C3, neither global nor local stimuli showed a processing advantage. In Condition C4, global RTs were as fast as local $\mathrm{RTs}$; however, the local interference became stronger than the reverse. In Condition $\mathrm{C} 5$ in which the contrast of the crosses was the same as that of the local arrows, a local precedence effect emerged. Local RTs were faster than global RTs, and the local interference effect was larger than the global interference effect. These results showed clearly that the relative advantage for global processing of hierarchical stimuli was gradually reduced as the contrast of the crosses increased. The ANOVA also showed that the effect of increasing the contrast of the background crosses on responses to global stimuli was larger than that on responses to local stimuli. These results indicate that weakening the grouping of local elements of hierarchical patterns eliminated the global advantage; thus, they provide strong evidence for the argument that grouping is crucial for the perception of global structure.

In addition, we found that local RTs were also slowed as a function of the contrast of the crosses, even though this effect on local RTs was smaller than that on global RTs. When the contrast of the crosses approached that of the local arrows, the subjects had to select an individual local arrow from not only the global structures of the target shapes but from the background crosses as well. When the discriminability of the crosses was equal to that of the local arrows, the process of local selection became difficult. Background crosses may also produce lateral masking on the local arrows (Podrouzek et al., 1992); thus, they may have resulted in slowed local RTs.

Another finding in this experiment was that the effect of increasing the contrast of the crosses reduced global interference and enhanced local interference. This is different from the effect of filtering low-spatial-frequency components (Lamb \& Yund, 1993, 1996), which only slows global RTs but does not affect global interference. Though it is not clear at which level of information processing the crosses affect the interference effect, our data suggest that the mechanisms involved in manipulation of the strength of perceptual grouping differ from the effects of low-spatial-frequency filtering.

\section{EXPERIMENT 3}

In Experiment 3, we also studied the effect of background crosses on the processing of global and local levels of stimuli, but we required discriminations based on closure of shapes. Previous studies have shown that the selection of local elements is more difficult when orientation discrimination, rather than closure discrimination, is required; hence, stronger global precedence effects emerge for orientation discrimination (Han et al., 1999a; see also Kimchi, 1994). Nevertheless, we expect that varying the contrast of the background elements will lead to a systematic weakening in the global precedence effect via slowing global RTs, though the "baseline" starting point (without background crosses) will differ relative to when orientation discrimination was required (Experiment 2). Furthermore, because of the ease of encoding closure (Chen, 1982b, 1985; Elder \& Zucker, 1993) and the reduced selection effort involved in the closure task relative to the orientation task (Han \& Humphreys, 1999; Han et al., 1999a), we expect a weaker effect of increasing the contrast of the background crosses on local responses when closure discrimination is required than when orientation discrimination is required.

\section{Method}

Subjects. Ten graduate students (all male; 22-32 years of age) from the University of Science and Technology of China partici-

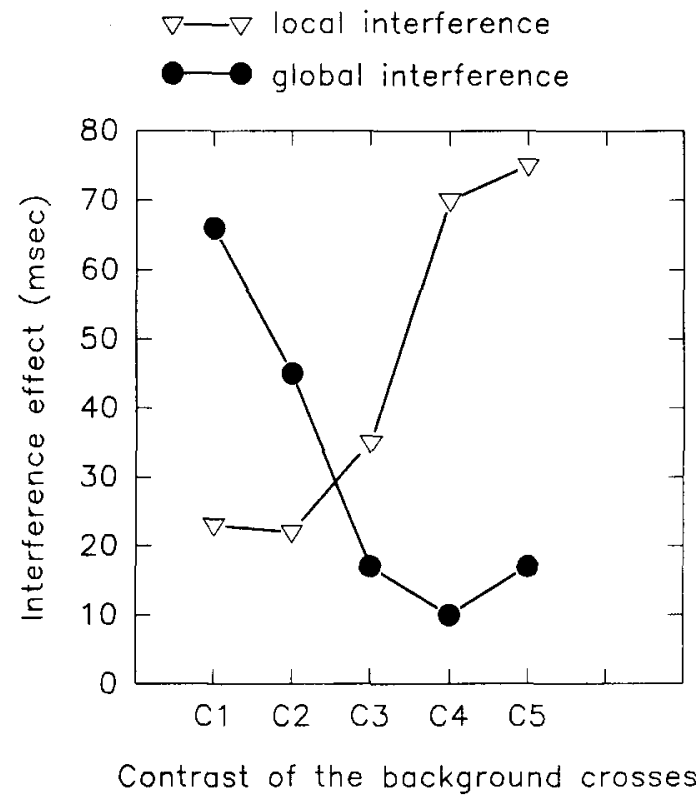

Figure 3. Interference effect (in milliseconds) as a function of the contrast of the background crosses in Experiment 2. Global interference $=$ local $R T s$ in the inconsistent conditions minus those in the consistent conditions. Local interference $=$ global $R T s$ in the inconsistent conditions minus those in the consistent conditions. 
pated in Experiment 3 as paid volunteers. All had normal or corrected-to-normal vision.

Apparatus, Stimuli, and Procedure. All aspects were the same as those in Experiment 2 except that the compound stimuli were global arrows or triangles made up of local arrows or triangles, illustrated in Figure 4. The subjects were asked to discriminate arrows or triangles at the global or local level, respectively. Because triangles are closed patterns and arrows are not, the feature required for the discrimination was closure. The subjects responded to global or local arrows by pressing one of two keys on a keyboard, and they responded to global or local triangles by pressing another key.

\section{Results}

The mean percentage errors for the globally and locally relevant tasks were $2.7 \%$ and $1.4 \%$, respectively. The mean error rates under each condition are given in Table 5.

There was a reliable main effect of contrast $[F(1,9)=$ $16.2, p<.001]$; error rates increased as the contrasts of the background crosses increased. The effect of globality was significant $[F(1,9)=11.10, p<.009]$, reflecting the fact that the subjects made more errors when responding to global stimuli than when responding to local stimuli. The effect of consistency $[F(1,9)=14.14, p<$ $.004]$ was also significant; error rates were higher when global and local shapes were inconsistent than when they were consistent. The contrast $\times$ globality interaction
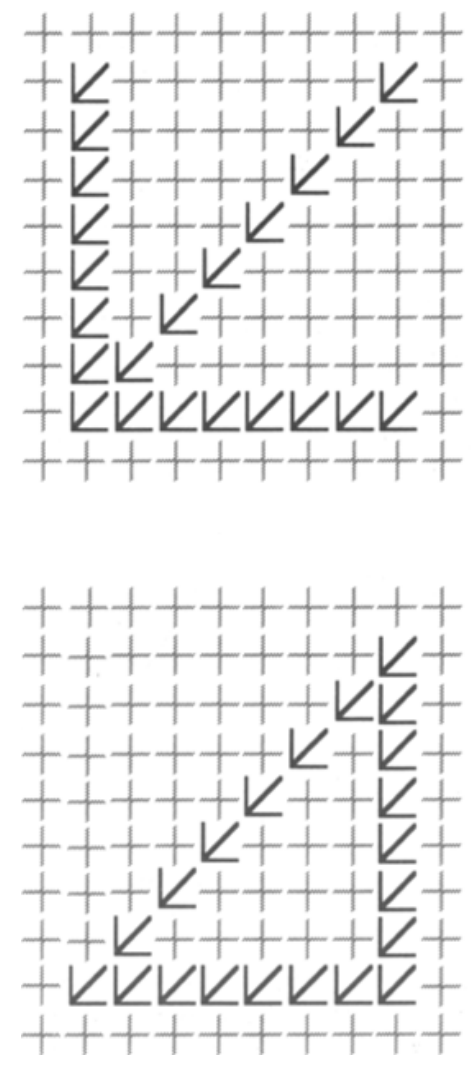

Figure 4. Compound stimuli used in Experiment 3. Global arrows or triangles consist of local arrows or triangles.

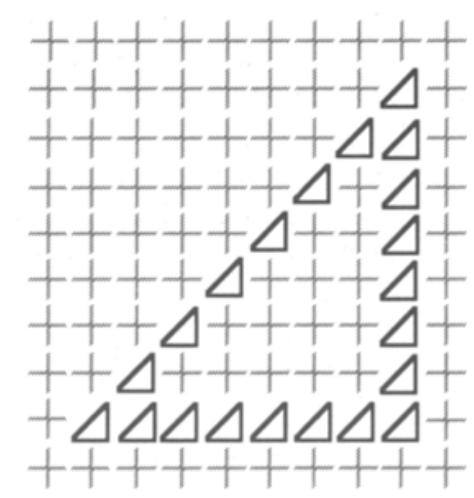

$[F(4,36)=3.95, p<.009]$ indicated that the effect of increasing the contrast of the crosses was stronger on global stimuli than on local stimuli. The effect of manipulating the contrast was also larger when global and local shapes were inconsistent than they were consistent, demonstrated by a reliable interaction between contrast and consistency $[F(3,36)=3.96, p<.009]$. The reliable globality $\times$ consistency interaction $[F(3,36)=5.45, p<.05]$ indicated that the effect of consistency was stronger on responses to global stimuli than on those to local stimuli. The triple interaction of the three factors was not significant $[F(3,36)=1.89, p>.1]$.

The mean RTs for the global and local stimuli are plotted in Table 6 as a function of the contrast of the background crosses. An ANOVA indicated a main effect of contrast $[F(4,36)=44.65, p<.0005]$; increasing the contrast of the crosses slowed the subjects' responses to the compound stimuli. There was also a main effect of globality $[F(1,9)=41.31, p<.0005]$, reflecting the fact that local RTs were faster than global RTs. The effect of consistency $[F(1,9)=147.54, p<.0005]$ was also reliable; RTs in the inconsistent condition were slower than those in the consistent condition.

The effect of increasing the contrast of the crosses on RTs was stronger on responses to the global stimuli than on those to the local stimuli, indicated by the contrast $X$

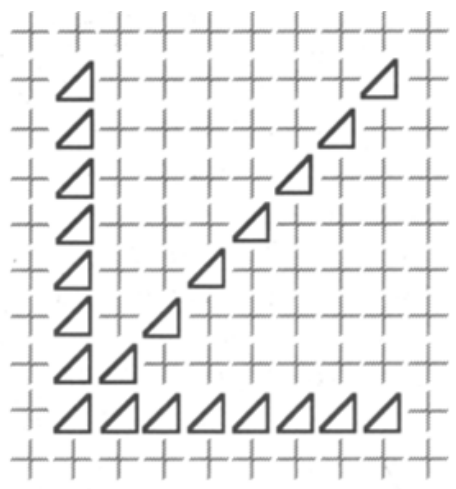


Table 5

Mean Percent Errors and Standard Errors Under Each Condition of Experiment 3

\begin{tabular}{|c|c|c|c|c|c|c|c|c|}
\hline \multirow{3}{*}{$\begin{array}{l}\text { Contrast } \\
\text { Condition }\end{array}$} & \multicolumn{4}{|c|}{ Global } & \multicolumn{4}{|c|}{ Local } \\
\hline & \multicolumn{2}{|c|}{ Consistent } & \multicolumn{2}{|c|}{ Inconsistent } & \multicolumn{2}{|c|}{ Consistent } & \multicolumn{2}{|c|}{ Inconsisten } \\
\hline & $M$ & $S E$ & $M$ & $S E$ & $M$ & $S E$ & $M$ & $S E$ \\
\hline $\mathrm{Cl}$ & 0.0 & 0.0 & 1.0 & 0.7 & 1.0 & 0.7 & 0.5 & 0.5 \\
\hline $\mathrm{C} 2$ & 1.4 & 1.0 & 1.0 & 0.7 & 1.4 & 1.0 & 2.0 & 0.8 \\
\hline C3 & 0.5 & 0.5 & 3.8 & 1.3 & 0.0 & 0.0 & 1.9 & 1.0 \\
\hline $\mathrm{C} 4$ & 2.0 & 0.8 & 6.5 & 1.0 & 1.0 & 0.7 & 3.8 & 1.1 \\
\hline $\mathrm{C} 5$ & 2.8 & 1.2 & 8.4 & 2.1 & 2.0 & 0.8 & 0.5 & 0.5 \\
\hline
\end{tabular}

globality interaction $[F(4,36)=42.41, p<.0005]$. Further orthogonal planned contrast tests demonstrated that increasing the contrast only decreased global RTs $[F(4,36)=56.57, p<.0005]$ but did not affect local RTs $[F(4,36)=1.07, p>.3]$. The globality $\times$ consistency interaction $[F(1,9)=11.24, p<.008]$ was significant; the interference effect was larger on responses to global stimuli than on those to the local stimuli. The consistency $\times$ contrast interaction was not significant $[F(4,36)=2.31$, $p>.05]$. Local interference on responses to global stimuli increased as a function of the contrast of the crosses (shown in Figure 5), indicated by the significant contrast $\times$ globality $\times$ consistency interaction $[F(4,36)=5.51$, $p<.001]$.

The data from Experiment 1 showed that the effect of increasing the contrast of the crosses differed according to whether local target elements were arrows or triangles. Accordingly, the global RTs in Experiment 3 were further analyzed with the factors of contrast of the crosses and forms of local figures (arrow vs. triangle). The ANOVA showed a main effect of the forms of the local figures $[F(1,9)=11.1, p<.009]$; responses to the global shapes made up of local triangles were faster than those to the global shapes made up of local arrows. However, the interaction between contrast and form was not significant $(p>.1)$; the effect of increasing the contrast of the crosses was similar for both types of global patterns composed of local arrows and triangles.

Separate analyses were also conducted for each contrast condition. There was no difference between global and local RTs for Conditions $\mathrm{C} 1(F<1)$ and $\mathrm{C} 2[F(1,9)=$ $1.13, p>.3$ ], but local RTs were faster than global RTs for Conditions C3 $[F(1,9)=56.73, p<.0005]$, C 4 $[F(1,9)=67.38, p<.0005]$, and C5 $[F(1,9)=89.42$, $p<.0005]$. The interference effect was significant under all the five contrast conditions $(p<.03)$. The globality $X$ consistency interaction was marginally significant $[F(1,9)=4.23, p<.07]$ for Condition $\mathrm{C} 1$. The interaction was not significant for Conditions $\mathrm{C} 2(F<1)$ and $\mathrm{C} 3[F(1,9)=1.31, p>.2]$, but it was significant for Conditions $\mathrm{C} 4[F(1,9)=15.43, p<.003]$ and $\mathrm{C} 5[F(1,9)=$ $6.56, p<.03]$. For Condition $\mathrm{Cl}$, there was no difference between global and local RTs, but global interference was stronger than local interference. No differences were found between global and local RTs, and there was no interference for Condition C2. There was a local prece- dence effect under Conditions C3, C4, and C5. Local RTs were faster than global RTs, and local interference was larger than the reverse. Global RTs slowed down as the contrast of the crosses increased and thus produced a stronger local precedence effect. RTs to local stimuli were unaffected.

\section{Discussion}

The data of Experiment 3 show that the effect of increasing the contrast of the background crosses was to slow global RTs. Local RTs, however, were surprisingly not affected. This effect led to variation of the relative priority for the local and global properties of hierarchical patterns as a function of the contrast of the background crosses. When there were no background elements, weak evidence for a global advantage occurred, since global interference was stronger than local interference, even though overall RTs did not differ. When the contrast of the crosses increased, the global RTs were slowed progressively and resulted in a complete local precedence effect. Overall RTs were faster to local stimuli, and local interference was stronger than the reverse (in Conditions $\mathrm{C} 3, \mathrm{C} 4$, and $\mathrm{C} 5$ ). These results are consistent with those of Experiment 2 and support our assertion that the background crosses eliminate the grouping of local elements into global stimuli. Due to this, there is a reduction in the global advantage. Furthermore, the fact that the background crosses did not affect local RTs in Experiment 3 but slowed local RTs in Experiment 2 gives strong support to the proposal that local elements were harder to select for orientation discrimination tasks than for closure discrimination tasks (Han et al., 1999a).

One difference between the results of Experiments 1 and 3 is that, in Experiment 3 (but not in Experiment 1), increasing the contrast of the crosses affected stimuli composed of local triangles. Trick and Enns (1997) demonstrated empirically Koffka's (1935) claim that there are two separable aspects of perceptual grouping: clustering (determining which elements belong together) and shape formation (determining cluster boundaries). They further showed that clustering spatial separate elements into countable entities does not demand attention, whereas attention is required for the process of shape formation of these elements. Similarly, both clustering and shape formation were necessary for recognizing the global shape for responses in our experiments. Local arrows and

Table 6

Mean Reaction Times (in Milliseconds) and Standard Errors Under Each Condition of Experiment 3

\begin{tabular}{|c|c|c|c|c|c|c|c|c|}
\hline \multirow{3}{*}{$\begin{array}{l}\text { Contrast } \\
\text { Condition }\end{array}$} & \multicolumn{4}{|c|}{ Global } & \multicolumn{4}{|c|}{ Local } \\
\hline & \multicolumn{2}{|c|}{ Consistent } & \multicolumn{2}{|c|}{ Inconsistent } & \multicolumn{2}{|c|}{ Consistent } & \multicolumn{2}{|c|}{ Inconsistent } \\
\hline & $M$ & $S E$ & $M$ & $S E$ & $M$ & $S E$ & $M$ & $S E$ \\
\hline $\mathrm{Cl}$ & 493 & 20.6 & 503 & 23.2 & 478 & 19.1 & 522 & 23.3 \\
\hline $\mathrm{C} 2$ & 503 & 23.1 & 519 & 23.1 & 494 & 22.3 & 506 & 21.7 \\
\hline $\mathrm{C} 3$ & 532 & 23.4 & 562 & 17.2 & 492 & 21.1 & 508 & 20.0 \\
\hline C4 & 546 & 21.5 & 614 & 21.8 & 494 & 19.9 & 510 & 21.2 \\
\hline C5 & 572 & 23.7 & 625 & 25.0 & 505 & 21.6 & 510 & 22.0 \\
\hline
\end{tabular}




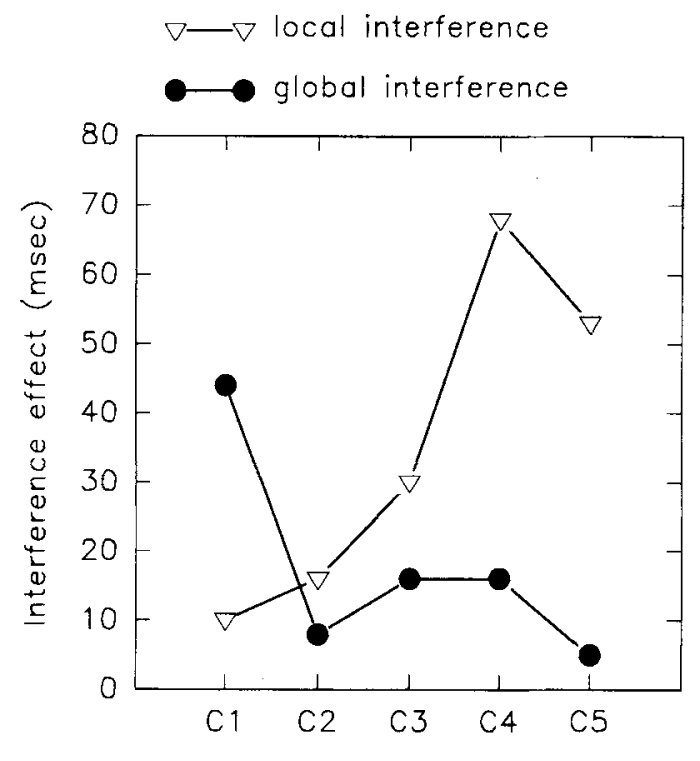

Contrast of the background crosses

Figure 5. Interference effect (in milliseconds) as a function of the contrast of the background crosses in Experiment 3. Global and local interference are computed in the same way as those in Experiment 2.

triangles should first be clustered into a unitary whole, and then the shapes of the whole used for responses. In Experiment 1, the local triangles grouped into several columns or rows (multiple perceptual wholes), and the subjects discriminated the orientations of these groups. In Experiment 2, however, all the local triangles grouped into one global arrow or triangle (one unitary whole), and the subjects discriminated whether the global shape was an arrow or a triangle. Therefore, there were fewer perceptual wholes for discrimination in Experiment 3 than in Experiment 1. Furthermore, the orientations of the parts of the global shapes were the same for the global arrow and triangle stimuli. Given these differences, it is possible that more attentional effort was involved for the discrimination task in Experiment 3 than in Experiment 1. This may be the case, especially when the contrast of the background crosses approached that of the local triangles (e.g., mean RTs in Conditions C4 and $\mathrm{C} 5$ in Experiment 3 were slower than those in Experiment 1). As a result, the perception of the global structures was impaired, even though the composing elements (triangles) differed from the crosses in closure.

In line with Experiment 2, global interference was reduced and local interference was enhanced as the contrast of the background elements increased in Experiment 3 .

\section{GENERAL DISCUSSION}

We first demonstrated that our new procedure of increasing the contrast of background figures eliminated grouping between local target elements. The effect of weakening grouping was significant only when local target elements differed from the background figures in orientation (Experiment 1). This procedure was then employed to examine further the role of perceptual grouping of local elements in hierarchical analysis. In general, the main effect of increasing the contrast of the crosses was to slow global RTs. This effect was also observed on local RTs in the orientation discrimination task in Experiment 2 (though the effect was much smaller). No effect was found on local RTs in the closure discrimination task in Experiment 3 . As a result, the global RT advantage was reduced and local RT advantages emerged. We also found that the interference effect showed a similar pattern of variation to the global RT advantage as a function of the contrast of the crosses. Systematically, global interference was reduced and local interference was enhanced when the contrast of the crosses was increased.

We suggest that local elements formed a strong group based on proximity when the compound stimuli were presented on a white background. When the contrasts of the background crosses and the compound stimuli were equated, global shapes could be perceived mainly from grouping by similarity of the shape. The degree to which grouping by similarity of shape was needed was increased systematically by increasing the contrasts of the background crosses. Because proximity dominates grouping at an earlier stage relative to similarity of shape (Ben-Av \& Sagi, 1995; Chen, 1982a, 1986; Han et al., 1999b), the transition from grouping by proximity to grouping by similarity of shape, which resulted from the increasing of the background crosses, retarded the perception of global shapes. As a consequence of this last effect, the perception of local features could dominate hierarchical analysis, and a local precedence effect resulted.

The present results fit well with a parallel processing model of hierarchical stimuli (Han et al., 1999a), in which perceptual grouping and the encoding of closure of local elements take place in parallel and compete with each other in determining the relative advantage of global and local levels of hierarchical stimuli. Decreasing the salience of the global shape, by decreasing the strength of grouping between local elements, leads to responses being slower for global shape information than for local shape information. This occurred directly as a function of the contrast of the background elements and irrespective of whether there was a strong or weak global precedence effect when no background elements were present. The ease of encoding closure (Chen, 1982b, 1985; Elder \& Zucker, 1993) and the reduced selection effort involved in the closure relative to the orientation task meant that responses to the closure of local elements were not affected by the background crosses; only RTs in the orientation discrimination task were slowed.

One might argue that the subjects adopted different attentional strategies across the various contrast conditions. For instance, the subjects might have attended to a single local arrow or triangle when performing the local task even when the contrast of the background crosses was increased. This strategy might have excluded the distract- 
ing crosses from the attentional focus, thus reducing their impact on local processing. When performing the global task, however, a large attentional focus may be required. This large focus could include the distracting crosses, with the results that there is impaired global processing. If this argument is correct and the different attentional strategies across the various contrast conditions were the dominant factors affecting the subjects' performance in the present study, we would expect RTs to the orientations of the perceptual groups to be slowed by increasing the contrast of the crosses no matter from which types of local elements (arrows and triangles) the groups were composed. This effect on local RTs would be also equivalent no matter which types of features (orientation or closure) were discriminated. However, the results of this study lend no support to this argument. Similar to other studies (Han, Fan, Chen, \& Zhuo, 1997; Lamb \& Yund, 1996), our data argue against the notion that selection between global and local levels of hierarchical patterns is based on the diameter of an attentional "spotlight."

A final note concerns a possible argument concerning variations in the spatial-frequency components of the stimuli. As we have already pointed out, the background crosses should not only attenuate grouping between local elements but they also introduce spatial-frequency noise. Studies have shown that the global advantage is decreased when subjects respond to compound stimuli devoid of low-spatial-frequency components (Badcock et al., 1990; Hughes et al., 1990; Lamb \& Robertson, 1990; Lamb \& Yund, 1993, 1996). The spatial-frequency hypothesis claims that the analysis of global and local levels of compound stimuli depends on the difference in spatial frequencies between local and global forms. Information at the local level is carried in relatively highspatial-frequency channels, whereas information at the global level is carried in relatively low-spatial-frequency channels. The global RT advantage reflects the temporal processing advantage provided by low spatial frequencies. It is possible here that spatial-frequency noise produced by the background crosses also contributed to the elimination of global precedence effect. However, the pattern of our present results goes beyond the predictions of the spatial-frequency account. For example, any spatialfrequency noise introduced by the background crosses should be exactly the same for the two types of stimuli used in Experiment 1 here. Nevertheless, we found that the crosses slowed RTs only to stimuli composed of local arrows and not to stimuli composed of local triangles. This difference strongly suggests that grouping rather than differences in spatial-frequency components determines RTs here. Furthermore, some stimuli overlapped across the experiments (e.g., the global arrow composed of local arrows appeared in both Experiment 2 and Experiment 3); despite this, the background crosses produced quite different effects on the relative advantages of global and local processing in the two experiments. There was a com- plete local precedence effect for Contrast Condition $\mathrm{C} 3$ in Experiment 3 (the closure discrimination task), whereas no difference was found between global and local processing for the same contrast condition in Experiment 2, though the spatial-frequency components were the same for the stimulus in both experiments. It is clear that the features used for discrimination, rather than the spatialfrequency components, determine the relative advantage of the global and local processing of stimuli in vision. Finally, unlike previous work that manipulated the spatialfrequency components of stimuli (Lamb \& Yund, 1993, 1996), we showed in the present study that manipulating the strength of perceptual grouping affected both the global RT advantage and the magnitude of global interference. Though it is not clear whether the same mechanism underlies both the RT advantage and the interference effect here, the covariation of the two effects reiterates that the effects of manipulating strength of grouping and the effect of variation in spatial-frequency components may be modulated by different mechanisms.

\section{REFERENCES}

Badcock, J. C., Whitworth, F. A., Badcock, D. R., \& Lovegrove, W. J (1990). Low-frequency filtering and the processing of localglobal stumuli. Perception, 19, 617-629.

BAYL.IS, G., \& DRIVER, J. (1993). Visual attention and objects: Evidence for hierarchical coding of location. Journal of Experimental Psychology Human Perception \& Performance, 19, 451-470.

BEN-Av, M. B., \& SAGl, D. (1995). Perceptual grouping by simılarity and proximity: Experimental results can be predicted by intensity autocorrelations. Vision Research, 35, 853-866.

CHEN, L. (1982a, November). Competing organization with several simultaneous factors. Paper presented at the annual meeting of the Psychonomic Society, Minneapolis, MN.

CHEN, L. (1982b). Topological structure in visual perception. Science, 218, 699-700.

CHEN, L. (1985). Topological structure in the perception of apparent motion. Perception, 14, 197-208.

C.HEN. L. (1986). Topological perception-A possible dark cloud over computational theory. In Q. X. Sen (Ed.), Essays on cognitive sciences (pp. 250-301). Shangha1: People's Press of Shanghai

ElDER, J., \& ZUCKER. S. (1993). The effect of contour closure on the rapid discrimination of 2-dimensional shapes. Vision Research, 33, $981-991$.

Enns, J. T., \& Kingstone, A. (1995). Access to global and local properties in visual search for compound stimuli. Psychological Science, 6, 283-291

GiNSBURG, A. P. (1986). Spatial filtering and visual perception. In K. R. Boff, L. Kaufman, \& J. P. Thomas (Eds.), Handbook of perception and human performance (chap. 34, pp. 1-71). New York Wiley.

Grice. G. R., Canham, L., \& Boroughs, J. M. (1983). Forest before trees? It depends where you look. Perception \& Psychophysics, 33 , $121-128$

Han, S , Fan, S., Chen, L., \& Zhuo, Y. (1997). On the different processing of wholes and parts: A psychophysiological study Joumal of Cognitive Neuroscience, 9, 686-697.

HAN, S., \& HUMPHREYS, G. W. (1999). Segmentation and selection contribute to the local processing in hierarchical analysis. Manuscript in preparation.

Han, S., Humphreys, G. W., \& Chen, L. (1999a). Parallel and competitive processes in hierarchical analysis. Perceptual grouping and encoding of closure. Journal of Experimental Psychology Human Perception \& Performance, 25, 1411-1432. 
HaN, S., Humphreys, G. W., \& CheN, L. (1999b). Uniform connectedness and classical Gestalt principles of perceptual groupıng. Perception \& Psychophysics, 61, 661-674.

HÜBNER, R. (1997). The effect of spatial frequency on global precedence and hemispheric differences. Perception \& Psychophysics, 59, 187-201.

HugheS, H. C., FENDrich, R., \& REUTER-LoREnZ, P. A. (1990). Global versus local processing in the absence of low spatial frequencies. Journal of Cognitive Neuroscience, 2, 272-282.

Humphreys, G., Olson, A., Romani, C., \& Riddoch, M. (1996). Competitive mechanisms of selection by object and space: A neuropsychological approach. In A. F. Kramer, M. G. H. Coles, \& G. D. Logan (Eds.), Converging operations in the study of visual selective attention (pp. 365-394). Washington, DC: APA.

Kahneman, D., \& HeniK, A. (1981). Perceptual organization and attention. In M. Kubovy \& J. R. Pomerantz (Eds.), Perceptual organization (pp. 181-211). Hillsdale, NJ: Erlbaum.

Kahneman, D., \& Treisman, A. (1984). Changing views of attention and automaticity. In R. Parasuraman \& D. R. Davies (Eds.), Varieties of attention (pp. 29-62). Orlando, FL: Academic Press.

KIMCHI, R. (1994). The role of wholistic/configural properties versus global properties in visual form perception. Perception, 23, 489-504.

KINCHLA, R. A., \& WOLFE, J. M. (1979). The order of visual processing: "Top-down," "bottom-up," or "middle-out." Perception \& Psychophysics, 25, 225-231.

KoFFKA, K. (1935). Principles of Gestalt psychology. New York: Harcourt Brace.

Kramer, A. F., \& JACOBSon, A. (1991). Perceptual organization and focused attention: The role of objects and proximity in visual processing. Perception \& Psychophysics, 50, 267-284.

Kurylo, D. D. (1997). Time course of perceptual grouping. Perception \& Psychophysics, 59, 142-147.

LAGASSE, L. (1993). Effects of good form and spatial frequency on global precedence. Perception \& Psychophysics, 53, 89-105.

LAMB, M. R., \& RoBERTSON, L. C. (1988). The processing of hierarchical stimuli: Effects of retinal locus, locational uncertainty, and stimulus identity. Perception \& Psychophysics, 44, 172-181.

LAmb, M. R., \& RoberTson, L. C. (1990). The effect of visual angle on global and local reaction times depends on the set of visual angles presented. Perception \& Psychophysics, 47, 489-496.

LAMB, M. R., \& YUND, E. W. (1993). The role of spatial frequency in the processing of herarchically organized stimuli. Perception \& Psychophysics, 54, 773-784.
LAMb, M. R., \& YUND, E. W. (1996). Spatial frequency and attention: Effect of level-, target-, and location-repetition on the processing of global and local forms. Perception \& Psychophysics, 58, 363-373

LUNA, D. (1993). Effects of exposure duration and eccentricity of global and local information on processing dominance. European Journal of Cognitive Psychology, 5, 183-200.

Luna, D., Marcos-Ruiz, R., \& MERino, J. M. (1995). Selective attention to global and local information: Effects of visual angle, exposure duration, and eccentricity on processing dominance. Visual Cognition, 2, 183-200.

MARTIN, M. (1979). Local and global processing: The role of sparsity. Memory \& Cognition, 7, 476-484.

May, J. G., Gutierrez, C., \& Harsin, C. A. (1995). The time-course of global precedence and consistency effects. International Journal of Neuroscience, 80, 237-245.

Moore, C. M., \& EGETH, H. (1997). Perception without attention: EvIdence of grouping under conditions of inattention. Journal of Experimental Psychology: Human Perception \& Performance, 23, 339352.

NAvON, D. (1977). Forest before trees: The precedence of global features in visual perception. Cognitive Psychology, 9, 353-383.

Navon, D. (1983). How many trees does it take to make a forest? Perception, 12, 239-254.

Navon, D., \& Norman, J. (1983). Does global precedence really depend on visual angle? Journal of Experimental Psychology: Human Perception \& Performance, 9, 955-965.

Paquet, L., \& Merikie, P. M. (1984). Global precedence: The effect of exposure duration. Canadian Journal of Psychology, 38, 45-53.

Podrouzek, K. W., Modigliani, V., \& Di Lollo, V. (1992). Lateral masking as a determınant of global dominance. Perception, 21, 705716.

Pomerantz, J. R. (1983). Global and local precedence: Selective attention in form and motion perception. Journal of Experimental Psychology: General, 112, 512-540.

TRICK, L. M., \& ENNS, J. T. (1997). Clusters precede shapes in perceptual organization. Psychological Science, 8, 124-129.

WerTHEIMER, M. (1923). Untersuchungen zur Lehre von der Gestalt: II [Principles of perceptual organization]. Psychologische Forschung, 4, 301-350.

(Manuscript received April 18, 1997; revision accepted for publication July 25,1998 .) 